\title{
Acción biocida de plantas de Piper tuberculatum Jacq. sobre Diatraea saccharalis (Lepidóptera, Pyralidae)
}

\section{Biocid action of Piper tuberculatum Jacq. against Diatraea saccharalis (Lepidóptera, Pyralidae)}

\author{
Gladys V. Soberón', Consuelo Rojas ${ }^{1}$, Jorge Saavedra², Massuo J. Kato ${ }^{3}$ y \\ Guillermo E. Delgado'
}

\begin{abstract}
${ }^{1}$ Facultad de Ciencias Biológicas, Universidad Nacional Pedro Ruiz Gallo, Ciudad Universitaria, Juan XXIII No 391, LambayequePERU.

Email: Guillermo Delgado guidelg2001@yahoo.es 2 Facultad de Agronomía, Universidad Nacional Pedro Ruiz Gallo.

${ }^{3}$ Instituto de Química, Universidade de São Paulo, CP 26077, 05508900 São Paulo, SP, Brasil.
\end{abstract}

Presentado: 27/01/2006 Aceptado: 13/07/2006

\section{Resumen}

En el presente trabajo evaluamos la acción biocida sobre larvas del III estadío de Diatraea saccharalis, usando extractos acuoso, diclorometano-metanol (DCM:MeOH, 2:1) y alcohólico (EtOH, 96\%) de hojas, tallos y espigas maduras (con frutos y semillas) de Piper tuberculatum, en larvas del III estadío. El método de inoculación del extracto, previamente eluido con agua destilada, fue de aplicación tópica en el mesotorax de las larvas. Solamente los extractos DCM:MeOH y EtOH de espigas maduras y extracto DCM:MeOH de plantas in vitro mostraron niveles significativos de mortalidad larval. El mayor efecto tóxico correspondió a extractos de espigas maduras respecto a plantas in vitro y a extracto EtOH respecto a extracto $\mathrm{DCM}: \mathrm{MeOH}$, tal como lo expresan los resultados de las concentraciones letales a $50 \%\left(\mathrm{CL}_{50}\right)$ y $90 \%\left(\mathrm{CL}_{90}\right)$, en $72 \mathrm{~h}$ de exposición. Así tenemos que en el caso de espigas maduras fue: $\mathrm{CL}_{50}\left(0,11 \mathrm{mg} / \mathrm{mL}\right.$ con EtOH y $0,16 \mathrm{mg} / \mathrm{mL}$ con DCM:MeOH) y $\mathrm{CL}_{90}$ $(0,35 \mathrm{mg} / \mathrm{mL}$ con EtOH y $0,55 \mathrm{mg} / \mathrm{mL}$ con $\mathrm{DCM}: \mathrm{MeOH})$; en el caso de plantas in vitro, unicamente con DCM:MeOH, fue: $\mathrm{CL}_{50} 0,39 \mathrm{mg} / \mathrm{mL}$ y $\mathrm{CL}_{90} 2,62 \mathrm{mg} / \mathrm{mL}$. Los resultados de las rectas valores probitos-mortalidad expresaron la misma tendencia.

Palabras claves: Concentración letal, extracto $\mathrm{DCM}: \mathrm{MeOH}$, extracto $\mathrm{EtOH}$, propagación in vitro, susceptibilidad larval

\section{Abstract}

The biocid action of DCM:MeOH (2:1), EtOH and aqueous extracts of leaves, stems and mature spikes (with fruits and seeds) of field plants and DCM:MeOH (2:1) extract of in vitro plants of Piper tuberculatum on III larval stage of Diatraea saccharalis was evaluated. The method was by inoculating the previously eluted extract with distillate water as topic applications on the larval mesothorax. Only $\mathrm{DCM}: \mathrm{MeOH}$ and EtOH extracts of mature spikes and DCM:MeOH extract of in vitro plants showed significant levels of larval mortality. The corresponding highest toxic effect was (a) for mature spikes respect to in vitro plants and (b) EtOH extract respect to DCM:MeOH extract, according to the results showed for the lethal concentration to 50\% $\left(\mathrm{LC}_{50}\right)$ and $90 \%\left(\mathrm{LC}_{90}\right)$, in 72 hours of exposure. Thus, in the case of mature spikes was: $\mathrm{LC}_{50} 0,11 \mathrm{mg} / \mathrm{mL}$ with $\mathrm{EtOH}$ and $0,16 \mathrm{mg} / \mathrm{mL}$ with $\mathrm{DCM}: \mathrm{MeOH}$ ) and $\mathrm{LC}_{90}(0,35 \mathrm{mg} / \mathrm{mL}$ with EtOH and $0,55 \mathrm{mg} / \mathrm{mL}$ with $\mathrm{DCM}: \mathrm{MeOH})$; and, in the case of in vitro plants, only with $\mathrm{DCM}: \mathrm{MeOH}$ extract, was: $\mathrm{LC}_{50} 0,39 \mathrm{mg} / \mathrm{mL}$ and $\mathrm{LC}_{90} 2,62 \mathrm{mg} / \mathrm{mL}$. The results of probit values-mortality lines showed the same tendency.

Keywords: Lethal concentration, DCM:MeOH (2:1) extract, EtOH extract, in vitro propagation, larvicidal susceptibility.

\section{Introducción}

D iatraea saocharalis (Lepidóptera, Pyralidae) («borer, cañero, barrenador o taladro de la caña de azúcar») es considerada entre las plagas de insectos más importantes que afectan diversos cultivos a nivel mundial. En el Perú, se estima que más del 30\% de la cosecha de maíz se pierde por el ataque de ambas plagas; asimismo, valores significativos de pérdidas también ocurren con otros cultivos como la caña de azúcar, el sorgo y diversas especies hortícolas (Sarmiento y Rázuri, 1978).

Diversos metabolitos secundarios han sido aislados de especies del género Piper destacando los lignanos, neolignanos, alcaloides, chalconas, kawapironas, flavonas, aceites esenciales, amidas, entre otros (Sengupta y Ray, 1987; Parmar et al., 1997). Piper tuberculatum, «matico», es ampliamente distribuida en América desde Brasil hasta México, de ella han sido aisladas e identificadas siete amidas extraídas de las semillas con D CM:MeO H, de las cuales dos resultaron amidas isobutílicas y cinco piperidínicas (Navickiene et al., 2000). Todas estas amidas fueron ensayadas individualmente contra Cladosporium sphaerospermum, resultando que la cantidad mínima requerida para inhibir el crecimiento del hongo se encontraba en el rango de 0,1 a $5 \mathrm{mg}$ (Navickiene et al., 2000) y contra C. dadosporioides en el rango 5 a $10 \mathrm{mg}$ (Silva et al., 2002).

El presente trabajo tuvo como objetivo evaluar la acción biociday los niveles de susceptibilidad de extractos DCM:MeOH (2:1), EtOH y acuoso de hojas, tallos y espigas maduras (con frutos y semillas) de plantas silvestres y extracto de DCM:MeOH (2:1) de plantas in vitro de Piper tuberculatum sobre larvas del III estadío del «borer» D iatraea saccharalis.

\section{Material y métodos Material biológico}

El material botánico estuvo constituido por hojas, tallos y espigas maduras (con frutos y semillas) de Piper tuberculatum Jacq. colectadas de plantas adultas a orillas del Río Cumbil, pro- 


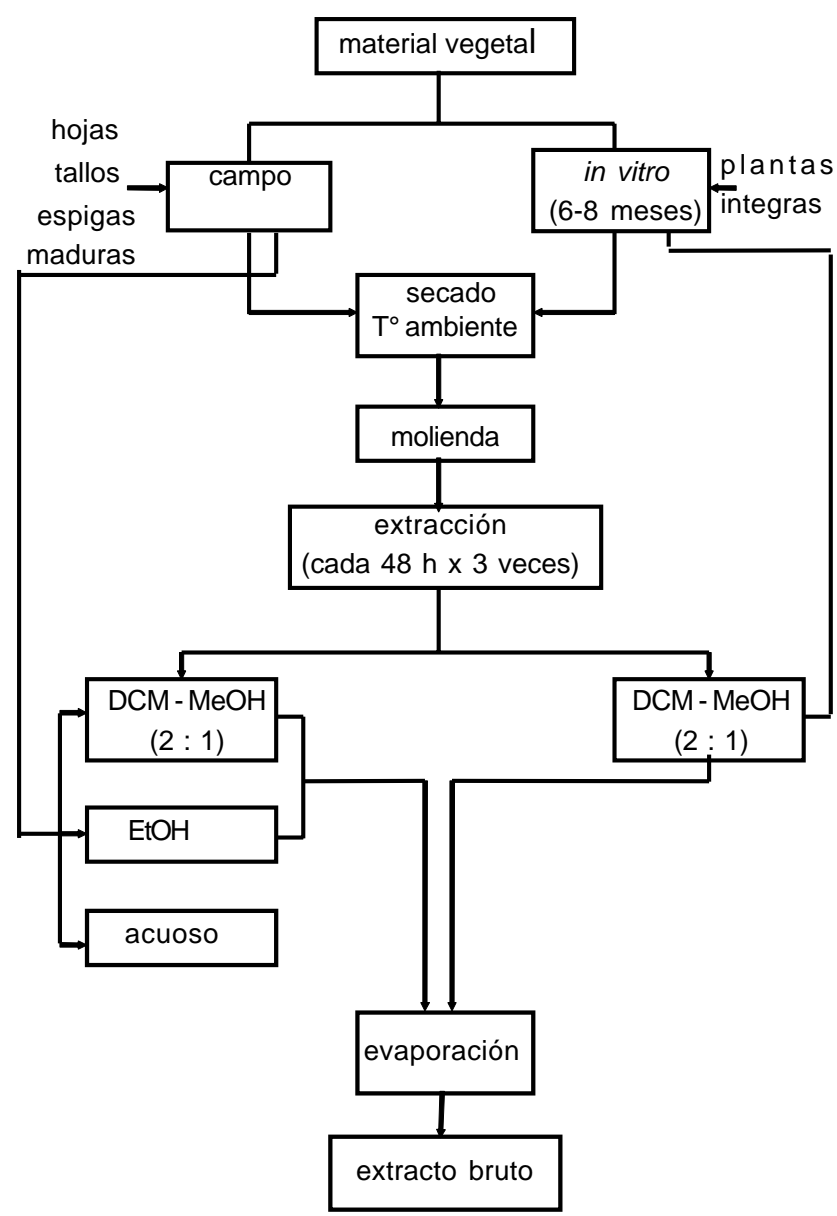

Figura 1. Procedimiento seguido en la obtención de extractos a partir de plantas silvestres e in vitro de $P$. tuberculatum.

vincia de Chota, región Cajamarca, entre los meses de agosto a noviembre del 2003, así como plantas in vitro crecidas en condiciones asépticas. Las plantas fueron identificadas por el Dr. G uillermo E. D elgado Paredes de la UNPRG, Lambayeque en base a la descripción realizada por Yuncker (1973). Una muestra herborizada fue depositada en el Herbario de la Universidad Nacional Pedro Ruiz G allo (UNPRG).

Los insectos-plaga de D iatraea sacharalis fueron colectados, en estadío de huevo, de cultivos de maíz del Fundo La Peña, Lambayeque, de propiedad de la Facultad de Agronomía de la UNPRG.

\section{Obtención y cultivo de plantas in vitro}

Las semillas de P. tuberculatum fueron desinfestadas con alcohol etílico 70\% durante 3 minutos e hipoclorito de sodio 5,25\% durante 5 minutos, luego enjuagadas con agua destilada esterilizada, e inoculadas en medio de cultivo conteniendo las sales minerales MS (Murashige y Skoog, 1962), las vitaminas minositol $100 \mathrm{mg} / \mathrm{L}$ y tiamina. $\mathrm{HCl} 1 \mathrm{mg} / \mathrm{L}$, sacarosa 2\% y agar 0,8\% ; realizándose esta actividad en el Laboratorio de Cultivo de Tejidos Vegetales y Recursos Genéticos de la Facultad de Ciencias Biológicas de la UNPRG. D espués de 3 meses de cultivo las plántulas fueron micropropagadas por ápices caulinaresy nudos, en medio de cultivo similar al anterior excepto que fue suplementado con ácido indol-3-acético (AIA) yácido giberélico $\left(\mathrm{AG}_{3}\right)$ 0,02 mg/ L, respectivamente. El pH del medio de cultivo fue ajustado en $5,7 \pm 0,1$ con $\mathrm{NaOH}$ y $\mathrm{HCl} 0,1 \mathrm{~N}$, respectivamente, y autoclavado a $15 \mathrm{lb} / \mathrm{pulg}^{2}$ de presión y $121^{\circ} \mathrm{C}$ de temperatura durante 20 minutos. La incubación de los cultivos se realizó con $10 \mathrm{~W} \cdot \mathrm{m}^{-2}$ de irradiancia, $24-26^{\circ} \mathrm{C}$ de temperaturay 16-8 $\mathrm{h}$ de fotoperiodo.

\section{Obtención e inoculación de larvas}

Las posturas de D . saccharalis fueron incubadas en $25 \pm 1^{\circ} \mathrm{C}$ de temperatura y $70 \pm 5 \%$ de humedad relativa en cámaras de vidrio. O currida la eclosión, las larvas fueron alimentadas con hojas y frutos frescos de maíz. Las pupas fueron sexadas, desinfestadas con hipoclorito de sodio 2,5\% durante 30 segundos y almacenadas en frascos de vidrio conteniendo algodones humedecidos y cubiertos con tul. Los adultos fueron almacenados en frascos de vidrio en proporción 4:5 (hembra:macho), donde se acondicionó maceteros con plántulas de maíz para facilitar la oviposición, y fueron alimentados con una solución de agua azucarada (agua y miel de abeja) en proporción 3:1, embebida en pequeñas tiras de papel de filtro. Con las posturas obtenidas se reinició el proceso hastala obtención de larvas en el III estadío y de la misma edad. Fueron instaladas 5 larvas por placa de Petri haciendo un total de 20 larvas por tratamiento. Los tratamientos fueron establecidos después de realizado bioensayos rápidos que determinaron el rango óptimo de efectividad del extracto; asimismo, cada tratamiento se repitió dos veces. El método de inoculación de las larvas fue el de aplicación tópica en el mesotorax, que consistió en aplicar la solución del extracto (previamente eluida con agua destilada) utilizando una micropipeta Eppendorf de $100 \mathrm{~mL}$ calibrada paraliberar $6,5 \mathrm{~mL}$ de la solución por larva con las dosis previamente definidas. Las larvas inoculadas fueron alimentadas con hojas y frutos frescos de maíz, realizándose las evaluaciones después de 24, 48 y 72 horas de la aplicación. Se asumió que una larva estaba muerta cuando, observada al estereomicroscopio, no reaccionaba al ser tocada en la región cervical con un puntero de punta roma. Las larvas testigo fueron inuculadas solamente con agua destilada.

\section{Preparación del extracto}

En el caso de plantas silvestres, hojas, tallos y espigas maduras fueron inicialmente secados al ambiente y luego en estufa a $50{ }^{\circ} \mathrm{C}$ durante una semana. Posteriormente, $45 \mathrm{~g}$ fueron molidos hasta obtener un polvo fino y sometidos a extracción, por separado, con diclorometano (D CM) - metanol (MeOH) 2:1 y alcohol etílico 96\% (EtOH) por tres veces consecutivas durante 48 horas. El extracto acuoso se obtuvo de manera directa hirviendo la muestra $(2,5 ; 5$ y $10 \mathrm{~g})$ por 10 minutos en $50 \mathrm{~mL}$ de agua destilada ( 5,10 y $20 \%$, respectivamente) hasta obtener un volumen final de $25 \mathrm{~mL}$; en el caso de plantas in vitro, plantas completas incluyendo hojas, tallos y raíces, de 6-8 meses de edad, fueron sometidas a un similar proceso de extracción pero utilizando D CM-MeOH (2:1) como único solvente de extracción (Figura 1).

\section{Análisis estadístico}

En el análisis estadístico se utilizó un diseño experimental de estímulo creciente donde los grupos experimentales estuvieron constituidos por larvas del III estadío de D. saccharalis a las que se aplicó como estímulo concentraciones crecientes de extractos D CM:MeOH, EtOH y acuoso de espigas maduras así como extracto D CM:MeOH de plantas in vitro de P. tuberculatum. 
Tabla 1. Rendimiento de extractos $\mathrm{DCM}: \mathrm{MeOH}$ y EtOH provenientes de plantas silvestres e in vitro de P. tuberculatum.

\begin{tabular}{lllccc}
\hline Procedencia & Explante & Solvente & $\begin{array}{c}\text { M asa seca } \\
\text { (g) }\end{array}$ & $\begin{array}{c}\text { Peso de } \\
\text { extracto }(\mathbf{g})\end{array}$ & $\begin{array}{c}\text { Rendimiento } \\
\text { (\%) }\end{array}$ \\
\hline Plantas de campo & Hoja & DCM:MeOH & 45 & 1,99 & 4,44 \\
& & EtOH & 45 & 2,26 & 5,02 \\
& \multirow{2}{*}{ Tallo } & DCM:MeOH & 45 & 0,71 & 1,58 \\
& \multirow{2}{*}{ Espiga madura } & EtOH & 45 & 0,96 & 2,13 \\
& & DCM:M $: \mathrm{MOH}$ & 45 & 2,72 & 6,04 \\
Plantas in vitro & & DCM:MeOH & 9,4 & 5,31 & 11,78 \\
\hline
\end{tabular}

Los parámetros estadísticos $\mathrm{CL}_{50}$ (concentración letal media) y $\mathrm{CL}_{90}$ (concentración letal noventa) y sus límites de confianza fueron determinados utilizando el software U.S. EPA Probit Analysis Program Ver 1.5; Environmental Monitoring Systems Laboratory, Cincinnati, $\mathrm{OH}$, USA. Con los valores de $\mathrm{CL}_{50} \mathrm{y}$ $\mathrm{CL}_{90}$ se trazaron las rectas de regresión log Concentración-Mortalidad Probit, utilizando papel semilogarítmico, colocando en el eje de las ordenadas los porcentajes de mortalidad en valores próbitos (5 y 6,28, respectivamente) obtenidos de la tabla de Fisher y Yates (Busvine, 1957), y en el eje de las abscisas las concentraciones letales.

\section{Resultados}

El mayor rendimiento porcentual de extractos de plantas silvestres de P. tuberculatum se alcanzó con espigas maduras y utilizando como solvente $\mathrm{EtOH}$; en plantas in vitro, el rendimiento fue similar al obtenido con espigas maduras y DCM:MeOH (Tabla 1). En ambos casos el rendimiento fue superior a lo observado en hojas y tallos de plantas silvestres.

Referente ala acción biocida de extractos, ensayos preliminares demostraron que los extractos D CM:MeO H, EtO H y acuoso de hojas y tallos de plantas silvestres así como el extracto acuoso de espigas maduras no ejercieron efecto alguno sobre larvas del III estadío de D . saccharalis, razón por la cual los resultados no son mostrados en tablas.

Una mortalidad de $100 \%$ fue alcanzada con extracto DCM:MeOH de espigas maduras a $48 \mathrm{~h}$ a $0,09 \mathrm{mg} / \mathrm{mL}$ de concentración (Tabla 2), en tanto que 95\% de mortalidad fue alcanzada con extracto EtO $\mathrm{H}$ de espigas maduras, también a 48 $\mathrm{h}$ pero a 0,18 mg/ mL de concentración (Tabla 3). Referente a plantas in vitro, cuyos extractos fueron obtenidos únicamente con D CM:MeO H, 85\% de mortalidad fue alcanzada a $48 \mathrm{~h}$ con $0,18 \mathrm{mg} / \mathrm{mL}$ de concentración (Tabla 4). En todos los casos la mortalidad del testigo fue cero por ciento.

En las tabla 5 se presentan los rangos de valores de concentraciones letales al 50\% y 90\% con sus límites de confianza y confiabilidad a 95\%, tanto para espigas maduras como para plantas in vitro. En el caso de espigas maduras, los extractos de EtOH alcanzaron un patrón de efectividad ligeramente superior respecto a los extractos D CM:MeOH, tanto para $\mathrm{CL}_{50}$ como para $\mathrm{CL}_{90}$, a $72 \mathrm{~h}$ de exposición. A sí, 50\% de mortalidad larval se alcanzó con EtO H 0,11 mg/ mL (0,08-0,15 mg/ mL) respecto a D CM:MeOH con 0,16 mg/ mL (0,18-0,24 mg/ mL), en tanto que $90 \%$ de mortalidad larval se alcanzó con EtO H 0,35 mg/ $\mathrm{mL}(0,23-0,88 \mathrm{mg} / \mathrm{mL})$ respecto a D CM:MeO H con 0,55 mg/ $\mathrm{mL}(0,33-1,82 \mathrm{mg} / \mathrm{mL})$. En el caso de plantas in vitro, el extracto de DCM:MEOH, a 72 h de exposición, alcanzó un patrón de efectividad para $\mathrm{CL}_{50}$ de 0,39 $\mathrm{mg} / \mathrm{mL}(0,27-0,63 \mathrm{mg} / \mathrm{mL})$, en tanto que para $\mathrm{CL}_{90}$ de 2,62 mg/ mL (1,33-9,96 mg/ mL).

Las líneas de regresión (Figuras 2 a-d), expresan la proporción de mortalidad larvaria en valores probitos por el logaritmo de las concentraciones $(\mathrm{mg} / \mathrm{mL}$ ) en $72 \mathrm{~h}$ de exposición al extracto correspondiente, tanto para $\mathrm{CL}_{50}$ como para $\mathrm{CL}_{90}$. En las figuras $2 \mathrm{a}-\mathrm{C}$, correspondientes a los extractos D CM:MeOH (Figura 2a) y EtO H (Figura 2b) de espigas maduras y D CM:MeOH (Figura 2c) de plantas in vitro, el patrón homogéneo de respuesta indica que, en todos los casos, la pendiente significa que por cada unidad de incremento en la concentración se produce un mayor incremento de mortalidad. Asimismo, en los extractos D CM:MeOH y EtOH de espigas maduras y DCM:MeOH de

Tabla 2. Porcentaje de mortalidad de larvas del III estadío de D. saccharalis con extracto DCM-MeOH de espigas maduras de $P$. tuberculatum.

\begin{tabular}{|c|c|c|c|c|c|c|}
\hline \multirow{2}{*}{$\begin{array}{l}\text { Concentración } \\
(\mathrm{mg} / 6,5 \mathrm{~mL})\end{array}$} & \multirow{2}{*}{$\begin{array}{c}\text { Concentración } \\
(\mathrm{mg} / \mathrm{mL})\end{array}$} & \multicolumn{3}{|c|}{ M ortalidad (\%) } & \multirow{2}{*}{$\begin{array}{c}\text { M ortalidad } \\
\text { O bservada } \\
(\%)\end{array}$} & \multirow{2}{*}{$\begin{array}{c}\text { Mortalidad } \\
\text { Corregida } \\
(\%)\end{array}$} \\
\hline & & $24 h$ & $48 h$ & $72 \mathrm{~h}$ & & \\
\hline 0,0 & 0 & 0 & 0 & 0 & 0 & 0 \\
\hline 0,005 & 0,0007 & 0 & 0 & 0 & 0 & 0 \\
\hline 0,009 & 0,0014 & 0 & 0 & 0 & 0 & 0 \\
\hline 0,019 & 0,0029 & 0 & 0 & 0 & 0 & 0 \\
\hline 0,038 & 0,0057 & 0 & 5 & 10 & 10 & 10 \\
\hline 0,075 & 0,0115 & 10 & 10 & 20 & 30 & 30 \\
\hline 0,15 & 0,0230 & 25 & 30 & 40 & 40 & 40 \\
\hline 0,3 & 0,0460 & 65 & 80 & 80 & 80 & 80 \\
\hline 0,6 & 0,0920 & 70 & 100 & 100 & 100 & 100 \\
\hline 1,2 & 0,1850 & 85 & 100 & 100 & 100 & 100 \\
\hline
\end{tabular}



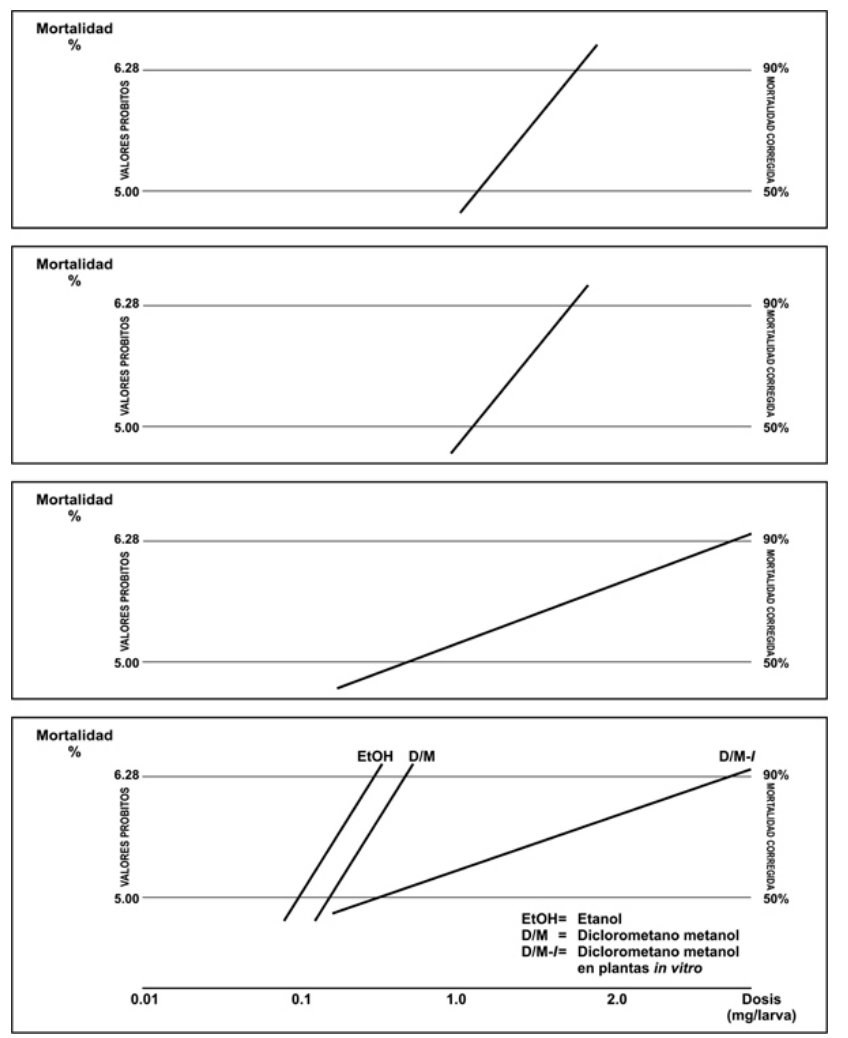

Figura 2 a-d. Líneas de regresión entre los valores probitos o mortalidad probit (\%) por el logaritmo de las concentraciones (Concentración: $\mathrm{mg} / \mathrm{mL}$ ) a 72 h de exposición en diferentes extractos de $P$. tuberculatum sobre el III estadío larval de $D$. saccharalis (a). Extracto DCM:MeOH de espigas maduras, (b). Extracto EtOH de espigas maduras, (c). Extracto DCM:MeOH de plantas in vitro, (d). Extracto EtOH y DCM:MeOH de espigas maduras y DCM:MeOH de plantas in vitro.

plantas in vitro, el valor de las pendientes de las líneas de regresión (Figura 2d), indica que los extractos EtO H y DCM:MeOH de espigas maduras fueron superiores al extracto DCM:MeOH de plantas in vitro, en especial para $\mathrm{CL}_{90}$.

\section{Discusión}

Tal como fue indicado anteriormente, los extractos DCM:MeOH, EtOH y acuoso de hojas y tallos de plantas silvestres así como el extracto acuoso de espigas maduras no ejercieron efecto significativo sobre larvas del III estadío de D. saccharalis, lo que puede atribuirse, en el caso de hojas y tallos, a una distribución restringida de las amidas y de otros compuestos con actividad insecticida; en el caso del extracto acuoso, sin duda no es un procedimiento de extracción adecuado. Por el contrario, los extractos de DCM:MeOH y EtOH de espigas maduras y DCM:MeO H de plantas in vitro, exhibieron una potente actividad insecticida sobre larvas del III estadío de ésta especie de lepidóptera. En efecto, tal como lo expresan los valores de $\mathrm{CL}_{50} \mathrm{yCL}_{90}$, los extractos D CM:MeO H y EtO $\mathrm{H}$ de espigas maduras exhibieron una mayor toxicidad que el extracto DCM:MeOH de plantas in vitro y el extracto EtOH exhibió, asimismo, una mayor efectividad que el extracto D CM:MeO H. Los resultados conllevan a sugerir dos posibilidades para su uso directo: en primer lugar, la eficiente actividad insecticida del extracto $\mathrm{EtOH}$ de espigas maduras de P. tuberculatum permite que el poblador rural utilice de manera amplia el extracto con aguardientes tradicionales como el «yonque» y el «cañazo», que son de fácil obtención, bajo costo y menor toxicidad que otros solventes orgánicos, y en segundo lugar, si bien es cierto que el extracto de plantas in vitro exhibió una menor toxicidad respecto a los extractos de espigas maduras, se presenta como una posibilidad para biosintetizar el principio activo a gran escala mediante el establecimiento de suspensiones celulares (D elgado, 1999; D anelutte et al., 2005).

Al respecto, numerosas especies de varias familias vegetales han sido utilizadas en el control de diversos insectos responsables en la transmisión de enfermedades o como plagas en todos los cultivos del mundo. Entre estas familias destaca la familia Piperaceae, especialmente los miembros del género Piper (Marquis, 1991; Bernard et al., 1995). Así tenemos que P. guinense y P. nigrum, son utilizados como insecticidas y moluscicidas en diferentes partes del Africa (Ivbijaro y Bolaji, 1990); las especies de la India P. longum, P. betle, P. peepuloides y P. cubeba han demostrado actividad insecticida contra mosquitos y moscas (Miyakado et al., 1989) y P. umbellatum, P. hispidum y P. auritum, nativas de América Central y el Noroeste de la Amazonia, son utilizados por las poblaciones indígenas para prevenir la malaria (Schultes, 1980). Algunas especies como P. aduncum, P. aequale, P. hispidum, P. reticulatum y P. tuberculatum que ocurren en el Perú, presentan significativa actividad en el control de mosquitos del género A edes (Bernard et al., 1995).

ReferenteaP. tuberculatum, $100 \mathrm{mg} / \mathrm{mL}$ de extracto hexánico de hoja indujo una mortalidad de 54\% en larvas de II estadío de

Tabla 3. Porcentaje de mortalidad de larvas del III estadío de D. saccharalis con extracto EtOH de espigas maduras de P. tuberculatum.

\begin{tabular}{|c|c|c|c|c|c|c|}
\hline \multirow{2}{*}{$\begin{array}{l}\text { Concentración } \\
(\mathrm{mg} / 6,5 \mathrm{~mL})\end{array}$} & \multirow{2}{*}{$\begin{array}{l}\text { Concentración } \\
(\mathrm{mg} / \mathrm{mL})\end{array}$} & \multicolumn{3}{|c|}{ M ortalidad (\%) } & \multirow{2}{*}{$\begin{array}{c}\text { M ortalidad } \\
\text { O bservada } \\
\text { (\%) }\end{array}$} & \multirow{2}{*}{$\begin{array}{c}\text { M ortalidad } \\
\text { Corregida } \\
(\%)\end{array}$} \\
\hline & & $24 \mathrm{~h}$ & $48 h$ & $72 \mathrm{~h}$ & & \\
\hline 0,0 & 0 & 0 & 0 & 0 & 0 & 0 \\
\hline 0,005 & 0,0007 & 0 & 0 & 0 & 0 & 0 \\
\hline 0,009 & 0,0014 & 5 & 0 & 5 & 5 & 5 \\
\hline 0,019 & 0,0029 & 5 & 0 & 5 & 5 & 5 \\
\hline 0,038 & 0,0057 & 15 & 15 & 15 & 20 & 20 \\
\hline 0,075 & 0,0115 & 25 & 30 & 30 & 35 & 35 \\
\hline 0,15 & 0,0230 & 60 & 65 & 70 & 70 & 70 \\
\hline 0,3 & 0,0460 & 80 & 85 & 85 & 85 & 85 \\
\hline 0,6 & 0,0920 & 80 & 85 & 85 & 85 & 85 \\
\hline 1,2 & 0,1850 & 85 & 95 & 95 & 95 & 95 \\
\hline
\end{tabular}


Tabla 4. Porcentaje de mortalidad de larvas del III estadío de D. saccharalis con extracto DCM-MeOH de plantas in vitro de P. tuberculatum.

\begin{tabular}{|c|c|c|c|c|c|c|}
\hline \multirow{2}{*}{$\begin{array}{l}\text { Concentración } \\
(\mathrm{mg} / 6,5 \mathrm{~mL})\end{array}$} & \multirow{2}{*}{$\begin{array}{c}\text { Concentración } \\
(\mathrm{mg} / \mathrm{mL})\end{array}$} & \multicolumn{3}{|c|}{ M ortalidad (\%) } & \multirow{2}{*}{$\begin{array}{c}\text { M ortalidad } \\
\text { O bservada } \\
(\%)\end{array}$} & \multirow{2}{*}{$\begin{array}{c}\text { M ortalidad } \\
\text { Corregida } \\
(\%)\end{array}$} \\
\hline & & $24 \mathrm{~h}$ & $48 h$ & $72 h$ & & \\
\hline 0,0 & 0 & 0 & 0 & 0 & 0 & 0 \\
\hline 0,005 & 0,0007 & 0 & 0 & 0 & 0 & 0 \\
\hline 0,009 & 0,0014 & 0 & 0 & 0 & 0 & 0 \\
\hline 0,019 & 0,0029 & 5 & 5 & 5 & 5 & 5 \\
\hline 0,038 & 0,0057 & 10 & 10 & 10 & 10 & 10 \\
\hline 0,075 & 0,0115 & 10 & 15 & 15 & 15 & 15 \\
\hline 0,15 & 0,0230 & 15 & 15 & 20 & 20 & 20 \\
\hline 0,3 & 0,0460 & 15 & 30 & 35 & 35 & 35 \\
\hline 0,6 & 0,0920 & 30 & 50 & 60 & 60 & 60 \\
\hline 1,2 & 0,1850 & 55 & 85 & 85 & 90 & 90 \\
\hline
\end{tabular}

A edes atropalpus, después de $24 \mathrm{~h}$ de exposición (Bernard et al., 1995); los autores atribuyeron la toxicidad del extracto a la ocurrencia de la amidaisobutílica 4,5-dihidropiperlongumina, puesto que $0,01 \mathrm{mg} / \mathrm{L}$ de la sustancia pura indujo una mortalidad larval de $47 \%$ en el mismo tiempo de exposición. En trabajos previos, realizados con extracto de semillas, fue demostrada la ocurrencia de dos amidas isobutílicas y cinco piperidínicas las que mostraron una fuerte actividad contra Cladosporium sphaerospermum, en especial piperina y 5,6dihidropiperlonguminina con 1 y $5 \mathrm{mg}$, respectivamente, como cantidad mínima requerida parainhibir el crecimiento del hongo (Navickiene et al., 2000); asimismo, la actividad antifúngica de varias amidas contra $C$. cladosporioides también fue observada utilizando como cantidad mínima inhibitoria 5 a $10 \mathrm{mg}$ (Silva et al., 2002). Estudios realizados con amidas de otras especies de Piperaceae como Piper hispidum (Navickiene et al., 2000) y P. arboreum (Silva et al., 2002) contra Cladosporium han arrojado resultados similares. Adicionalmente, flavononas e hidroquinonas preniladas de P. crassinervium (D anelutte et al., 2003) y cromenes de Peperomia villipetiola (Malquichagua et al., 2005), ensayados contra ambas especies de Cladosporium, mostraron, asimismo, una fuerte actividad antifúngica.

Por otro lado, comparando los resultados obtenidos por Bernard et al., (1995), en nuestro trabajo fue demostrado que concentraciones tan bajas como $0,01-1,8 \mathrm{mg} / \mathrm{mL}$, para extracto de espigas maduras y $0,2-1,8 \mathrm{mg} / \mathrm{mL}$, para plantas in vitro, indujeron una mortalidad superior a 50\% del III estadío larval de D. sacharalis, en $24 \mathrm{~h}$ de exposición. Este hecho tiene una profunda significación ecológicapuesto que presupone una ventaja el uso de extractos vegetales como fuente de una complejidad de moléculas que muestran diversas bioactividades, elevan- do los niveles de toxicidad en relación a los compuestos individuales químicamente puros, a lo que se suma el riesgo de inducir resistencia (Bobadilla et al., 2005). Como es conocido, la fitoquímica del género Piper ha revelado la ocurrencia de diversos compuestos como alcaloides, fenilpropanoides, lignanos, neolignanos, terpenos, flavononas, entre otros (Parmar et al., 1997), muchos de las cuales sinergizan insecticidas naturales y sintéticos (Bernard et al., 1995), por ejemplo, el fenilpropanoide dillapiol sinergiza no solamente con las piretrinas sino también con carbamatos y organocloratos (Parmary Tomar, 1983). Recientemente, ha sido propuesta como estrategia de trabajo el uso de extractos heterogéneos de toda la biomasa de la planta, para inducir un efecto sinergístico sobre algún organismo específico (Leatemia e Isman, 2004).

Se conoce muy poco sobre cómo estaría operando la 4,5dihidropiperlonguminina y otros compuestos relacionados sobre el III estadío larval de D. sacharalis; sin embargo, se atribuye su toxicidad a la ocurrencia en su estructura molecular del anillo metilenedioxifenil (MDP) (Bernard et al., 1995), tal como ha sido reportado para otros compuestos de estructura química similar como pipercida, guineensinamida, guineensina, pellitorina y kalecida, aisladas de P. guineensey muy activas en el control de adultos de M usca domestica (G bewonyo et al., 1993). 4,5-dihidropiperlonguminina, al igual que otras isobutilamidas alquil y olefínicas, se encuentra restringida a miembros de muy pocas familias de gran presencia en los trópicos húmedos como son las Piperaceae, Asteraceae y Rutaceae, donde la herbivoría es una potente fuerza selectiva. Las isobutilamidas aisladas en Piperaceae tienen bajo peso molecular, contienen un único átomo de nitrógeno y se presume que no sería «costosa» su biosíntesis (G reger, 1988).

Tabla 5. Concentraciones letales a $50 \%\left(\mathrm{CL}_{50}\right)$ y $90 \%\left(\mathrm{CL}_{50}\right)$, límites de confianza $(\mathrm{LC})$ y pendientes (b) de extractos de $\mathrm{DCM}-\mathrm{MeOH}$ y EtOH de espigas maduras de $P$. tuberculatum sobre larvas del III estadío de $D$. saccharalis en $72 \mathrm{~h}$ de exposición.

\begin{tabular}{|c|c|c|c|c|c|c|}
\hline \multirow[t]{2}{*}{ Especie } & \multirow[t]{2}{*}{ Solvente } & \multicolumn{4}{|c|}{ Concentraciones Letales $(\mathrm{mg} / \mathrm{mL})$} & \multirow[t]{2}{*}{$\mathbf{b}$} \\
\hline & & $\mathrm{CL}_{50}$ & LC & $\mathrm{CL}_{90}$ & LC & \\
\hline Espigas maduras & $\begin{array}{l}\text { DCM-MeOH } \\
\text { EtOH }\end{array}$ & $\begin{array}{l}0,16 \\
0,11\end{array}$ & $\begin{array}{l}0,18-0,24 \\
0,08-0,15\end{array}$ & $\begin{array}{l}0,55 \\
0,35\end{array}$ & $\begin{array}{l}0,33-1,82 \\
0,23-0,88\end{array}$ & $\begin{array}{l}2,4 \\
2,5\end{array}$ \\
\hline Plantas in vitro & $\mathrm{DCM}-\mathrm{MeOH}$ & 0,39 & $0,27-0,63$ & 2,62 & $1,33-9,96$ & 1,6 \\
\hline
\end{tabular}




\section{Literatura citada}

Bernard, C.B., H.G. Krishnamurty, D. Chauret, T. Durst, B.J.R. Philogène, I. Sánchez-Vindas, C. Hasbun, L. Poveda, L. San Román \& J.T. Arnason. 1995. Insecticidal defenses of Piperaceae from the neotropics. J. Chem. Ecol. 21(6):801-814.

Bobadilla, M., F. Zavala, M. Sisniegas, G. Zavaleta, J. Mostacero \& L. Taramona. 2005. Evaluación larvicida de suspensiones acuosas de Annona muricata Linnaeus «guanabana» sobre Aedes aegypti Linnaeus (Diptera, Culicidae). Rev. Peruana Biol. 12(1):145-152.

Busvine, J.R. 1957. A Critical Review of the Techniques for Testing Insecticides. pp. 267-268. 2da. Edición. Commonwealth Agriculture Bureaux. London.

Danelutte, A.P., J.H.G. Lago, M.C.M. Young \& M.J. Kato. 2003. Antifungal flavonones and prenylated hydroquinones from Piper crassinervium Kinth. Phytochemistry 64:555559.

Danelutte, A.P., M.B. Costantin, G.E. Delgado, R. Braz-Filho \& M.J. Kato. 2005. Divergence of secondary metabolism in cell suspension cultures and differentiated plants of Piper cernuum and P. crassinervium. J. Braz. Chem. Soc. 16(6B):1425-1430.

Delgado, G.E. 1999. Metabólitos Secundários em Cultura de Tecidos de Piperaceae. Relatório Annual. Projeto de PósDoutoramento IQ-USP/FAPESP. Universidade de São Paulo, Brasil.

Gbewonyo, W.S.K., D.J. Candy \& M. Anderson. 1993. Structureactivity relationships of insecticidal amides from Piper guineense root. Pestic. Sci. 37:57-66.

Greger, H. 1988. Comparative phytochemistry of the alkamides. pp. 159-178. In: J. Lam, H. Breteler, T. Arnason \& 1. Hansen (eds.). Chemistry and Biology of Naturally Occurring Acetylenes and Related Compounds. Bioactive Molecules. Vol. 7. Elsevier, Amsterdam.

Ivbijaro, M.F. \& O.O. Bolaji. 1990. Effects of cypermethrin + dimethoate and extracts of $\mathrm{P}$. guineense and Azadirachta indica on the pests and yield of cowpea, Vigna unguiculata. J. Agric. Sci. 115:227-231.

Leatemia, J. \& B. Isman. 2004. Toxicity and antifeedant activity of crude seed extracts of Annona squamosa (Annonaceae) against lepidopteran pests and natural enemies. Int. J. Trop. Insect Sci. 24(1):150-158.
Malquichagua, K.J., G.E. Delgado, L. Ripalda, M.C.M Young \& M.J. Kato. 2005. Chromenes of polyketide origin from Peperomia villipetiola. Phytochemistry 66:573-579.

Marquis, R.J. 1991. Herbivore fauna of Piper (Piperaceae) in a Costa Rican wet forest: Diversity, specificity, and impact. pp. 177-199. In: P.W. Price, T.M. Lewinsohn, G.W. Fernandes \& W.W. Benson (eds.). Plant-Animal Interactions: Evolutionary Ecology in Tropical and Temperate Regions.

Miyakado, M., I. Nakayama \& N. Ohno. 1989. Insecticidal unsaturated isobutylamides: From natural products to agrochemical leads. pp. 183-187. In: J.T. Arnason, B.J.R. Philogène \& P. Morand (eds.). Insecticides of Plant Origin. ACS Symposium Series 387, American Chemical Society, New York.

Murashige, T. \& Skoog, F. 1962. A revised medium for rapid growth an bioassays with tissue culture. Physiol. Plant. 15:473497.

Navickiene, H.M.D., A.C. Alécio, M.J. Kato, V. da S. Bolzani, M.C.M. Young, A.J. Cavalheiro \& M. Furlan. 2000b. Antifungal amides from Piper hispidum and Piper tuberculatum. Phytochemistry 55:621-626.

Parmar, B.S. \& S.S. Tomar. 1983. Review of research on insecticide synergists in India - retrospect and prospect. Int. J. Trop. Agric. 1(1):7-17.

Parmar, V.S., S.C. Jain, K.S. Bisht, R. Jain, P. Taneja, A. Jha, O.M. Tyagi, A.K. Prasad, J. Wengel, C.E. Olsen \& P.M. Boll. Phytochemistry of the genus Piper. Phytochemistry 46:597-673.

Sarmiento J.M. \& R.V. Razuri. 1978. Bacillus thuringiensis en el control de Spodoptera frugiperda y Diatraea saccharalis en maíz. Rev. Peruana Entomología 21:121-124.

Schultes, R.F. 1980. De Plantis Toxicariis e Mundo Novo Commentationes XXVI. Ethnopharmacological notes on the flora of northwestern South America. Bot. Mus. Leafl. Harv. Univ. 28(1):1-45.

Sengupta, S. \& A.B. Ray. 1987. The chemistry of Piper species: a review. Fitoterapia LVIII (3):147-165.

Silva, R.V., H.M.D. Navickiene, M.J. Kato, V.S. Bolzani, C.I. Méda, M.C.M. Young \& M. Furlán. 2002. Antifungal amides from Piper arboreum and Piper tuberculatum. Phytochemistry 59:521-527.

Yuncker, T.G. 1973. The Piperaceae of Brazil II: Piper - Group V; Ottonia, Pothomorphe; Sarcorhachis. Hoehnea 3:29-284. 